\title{
ON A THEORY OF LINEAR DIFFERENTIAL EQUATIONS IN
}

\section{GENERAL ANALYSIS*}

BY

\section{T. H. HILDEBRANDT}

The fundamental principles of generalization have been well formulated by E. H. Moore in the statement:

"The existence of analogies between central features of various theories implies the existence of a general theory which underlies the particular theories and unifies them with respect to those central features."

In applying this principle to the analogies between the theory of linear integral equations and the theory of systems of linear algebraic equations and biquadratic forms, Moore was led to the construction of a body of General Analysis, consisting of a theory of properties of classes of functions on a general range, developed in his Introduction to general analysis; $\dagger$ a theory of functional operations, sketched in the papers, On a form of general analysis with applications to linear differential and integral equations, $\ddagger$ and $O n$ the fundamental functional opcration of a general theory of linear integral equations; $\$$ a general theory of linear integral equations, outlined in the paper on Foundations of the theory of linear integral equations $\|$ and the paper on Functional operations; and an existence theorem for general differential equations. $\uparrow$

It stands to reason that these general theories, in addition to the wide range of results obtainable as a result of specialization, may also serve as a basis for the consideration of generalizations in analogous fields. The present paper is an application of some of the concepts of the General Analysis and some of the results of the general theory of integral equations to the theory of general linear differential equations. The possibility of such an application

* The first part of a paper presented to the Society, December 31, 1915.

$\dagger$ Yale Colloquium Lectures (1910), pp. 1-149. Referred to as "General Analysis." An excellent introduction to the General Analysis is contained in O. Bolza's Einfuehrung in $E$. $H$. Moore's General Analysis. J a hresbericht der Deuts chen M athematiker Vereinigung, vol. 23 (1914), pp. 248-303.

$\ddagger$ Atti di IV Congresso (Rome) (1908), vol. II, pp. 98-114.

$\S$ Proceedings of the Fifth International Congress (Cambridge), vol. I, pp. 230-255. Referred to as "Functional Operations."

\|B Bulletin of the American Mathematical Society, vol. 18 (1912), pp. 334-362. Referred to as "Integral Equations."

I See Rome Memoir ( $\ddagger$ ) and lectures at the University of Chicago. 
was suggested by the results obtained by Moore in the derivation of existence theorems for general differential equations. The path to be pursued was pointed out by L. Schlesinger in his paper: Zur Theorie der linearen IntegroDifferentialgleichungen, ${ }^{*}$ who observes the analogy between systems of linear differential equations, and integro-differential equations of the form:

$$
\frac{d}{d z} \eta(p, q ; z)=\alpha(p, q ; z)+\int_{a}^{b} \alpha(p, r ; z) \eta(r, q ; z) d r,
$$

where $p$ and $q$ are continuous real variables, and $z$ ranges over a complex field, while $\eta$ and $\alpha$ are continuous in $p$ and $q$, and analytic in $z$. We shall consider a general theory based on an equation analogous to this equation, $p$ and $q$ being replaced by general variables, the condition of continuity of the functions $\alpha$ and $\eta$, by that of belonging to certain given classes, and integration by a general operator $J$. Instead of allowing $z$, however, to vary over a region in the complex plane, and the functions $\eta$ and $\alpha$ to be analytic in $z$, we restrict ourselves to the simpler case where $z$ is the real variable $x$ on a finite linear interval and the functions $\eta$ and $\alpha$ are continuous in $x$.

In a later paper we shall make a study of the solutions of the general linear differential equations considered in this paper, which satisfy certain linear boundary conditions.

\section{The foundations}

For the sake of convenience and clearness, we collect in these preliminary paragraphs, those of the fundamental concepts and propositions developed by Moore in his General Analysis, which we shall find useful in the sequel.

1. The fundamental classes. $\dagger$ The element of generality is secured in our theory by the introduction of a general, absolutely unconditioned class $\mathfrak{P}$ of elements $p$. The class $\mathfrak{B}$ enters the theory mainly through the medium of functions $\mu(p)$ or $\mu$, which define for every element $p$ a real or complex number. An assemblage or class (Menge) of such functions will be denoted by $\mathfrak{M}$. If $\mathfrak{A}$ is the class of all real, or more generally, all complex numbers $a$, we shall use the terminology: $\mathfrak{M}$ is a class of functions on $\mathfrak{P}$ to $\mathfrak{A}$.

In addition to the general class $\mathfrak{B}$ and the class of functions $\mathfrak{M}$, we shall need the particular class $\mathfrak{X}$, a class of elements $x$ ranging over the linear interval $a \leqq x \leqq b$; and the class $\mathfrak{S}$ of all continuous functions on $\mathfrak{X}$ to $\mathfrak{A}$.

2. Properties of classes of functions. (a) Linear Extension and Linearity. $\ddagger$ If we construct all possible linear combinations $\sum_{i=1}^{n} a_{i} \mu_{i}$ of functions of the class $\mathfrak{M}$, we obtain, in general, a new class which is called the linear extension of the class $\mathfrak{M}$ and is denoted by the symbol $\mathfrak{M}_{L}$. In case the linear extension of a class is the class itself, then the class is said to be linear $(L): \mathfrak{M}^{L}$.

* Jahresbericht der Deutschen Mathematiker Vereinigung, vol. 24 (1915), pp. 84-125.

$\dagger$ Cf. E. H. Moore, General Analysis, pp. 24, 25.

$\ddagger$ Cf. E. H. Moore, loc. cit., pp. 36, 37. 
(b) Relatively Uniform Convergence. Closure of a Class.* The sequence of functions $\mu_{n}$ is said to converge to the function $\mu$ on $\mathfrak{P}$ relatively uniformly as to the scale $\sigma\left(L_{n} \mu_{n}=\mu(\mathfrak{P} ; \sigma)\right)$ in case for every $e$ there exists an $n_{c}$ such that if $n \geqq n_{e}$ we have

$$
\left|\mu_{n}-\mu\right| \leqq e|\sigma|
$$

identically for every $p$ of the class $\mathfrak{B}$. If for a class $\mathfrak{M}$ we take the limits of all sequences which converge relatively uniformly as to functions of a scale class $\mathfrak{S}$, we obtain a new class, called the extension of $\mathfrak{M}$ relatively to $\mathfrak{S}$ and denoted by $\mathfrak{M}_{\mathscr{E}}$. In case the extension of $\mathfrak{M}$ relative to itself is the original class, then $\mathfrak{M}$ is said to be closed $(C)$, i. e., $\mathfrak{M}^{c}$ is the same as $\mathfrak{M}_{\mathfrak{M}}=\mathfrak{M}$.

(c) Dominance. Dominance Properties $D$ and $D_{0} \cdot \dagger$ A function $\mu_{1}$ is said to dominate $\mu_{2}$ on $\mathfrak{B}$, in case we have for every $p$ of $\mathfrak{P}$

$$
\left|\mu_{2}(p)\right| \leqq\left|\mu_{1}(p)\right|
$$

The importance of dominance in the theory is due to the presence of the scale function in the inequality defining relative uniformity of convergence. The desirability of a nowhere negative real-valued scale function, and also of a single scale function to replace a sequence of such functions leads to the following two dominance properties or conditions on the class $\mathfrak{M}: \mathfrak{M}$ is said to have the dominance property $D_{0}\left(\mathfrak{M}^{D_{0}}\right)$ in case there is for every function $\mu$ of the class $\mathfrak{M}$ a nowhere negative real-valued dominating function $\mu_{0}$ of the class $\mathfrak{M}$; further $\mathfrak{M}$ is said to have the dominance property $D\left(\mathfrak{M}^{D}\right)$ in case for every sequence of functions $\mu_{n}$ of the class $\mathfrak{M}$ there exists a sequence of real numbers $a_{n}$ and a function $\mu$ of the class $\mathfrak{M}$ such that for every $n, a_{n} \mu$ dominates $\mu_{n}$, i. e.,

$$
\left|\mu_{n}\right| \leqq\left|a_{n} \mu\right| \text {. }
$$

(d) *-Extension. $\ddagger$ By a combination of linear extension and extension relative to a class, we obtain a new extension which plays a central rôle in the theory, viz., $\mathfrak{M}_{*}=\left(\mathfrak{M}_{L}\right)_{\mathfrak{M}}$. If $\mathfrak{M}$ has the property $D$, then this new class has the properties: $L, C$, and $D$.

3. Composition of classes. $\S$ Suppose we have two general classes $\mathfrak{P}^{\prime}$ and $\mathfrak{P}^{\prime \prime}$ of elements $p^{\prime}$ and $p^{\prime \prime}$ respectively. Then the composite class $\mathfrak{B}^{\prime} \mathfrak{B}^{\prime \prime}$ is the class of all pairs of elements $\left(p^{\prime}, p^{\prime \prime}\right)$ or $p^{\prime} p^{\prime \prime}$. Similarly if $\mathfrak{M}^{\prime}$ and $\mathfrak{M}^{\prime \prime}$ are classes of functions $\mu^{\prime}$ and $\mu^{\prime \prime}$ on $\mathfrak{P}^{\prime}$ and $\mathfrak{P}^{\prime \prime}$ respectively, to $\mathfrak{A}$, then the class $\mathfrak{M}^{\prime} \mathfrak{M} \mathfrak{M}^{\prime \prime}$ on $\mathfrak{B}^{\prime} \mathfrak{B}^{\prime \prime}$ is defined to consist of the products of all functions $\mu^{\prime}$ of $\mathfrak{M}^{\prime}$ by functions $\mu^{\prime \prime}$ of $\mathfrak{M}^{\prime \prime}$. The *-extension of $\mathfrak{M}^{\prime} \mathfrak{M}^{\prime \prime}$,

$$
\left(\mathfrak{M}^{\prime} \mathfrak{M}^{\prime \prime}\right)_{*}=\left(\left(\mathfrak{M}^{\prime} \mathfrak{M} \mathfrak{M}^{\prime \prime}\right)_{L}\right)_{\mathfrak{M}^{\prime}} \mathfrak{W}^{\prime \prime} \text {, }
$$

* Cf. E. H. Moore, loc. cit., pp. 29-37.

$\dagger$ Cf. E. H. Moore, loc. cit., pp. 39-42.

$\ddagger$ Cf. E. H. Moore, loc. cit., pp. 78-79.

$\S$ Cf. E. H. Moore, loc. cit., $\S \S 53,54,55$, pp. $93-96$ 
is called the *-composite of $\mathfrak{M}^{\prime}$ and $\mathfrak{M}^{\prime \prime}$, and is of special importance in the theory. We shall denote this class by $\Omega$ and the elements of the class by $\kappa\left(p^{\prime} p^{\prime \prime}\right)$ or $\kappa$. The class obtained by reversing the order of the elements $p^{\prime}$ and $p^{\prime \prime}$, i. e., $\left(\mathfrak{M}^{\prime \prime} \mathfrak{M}^{\prime}\right)$ * will be denoted by $\breve{\Omega}(\Omega$-transposed) and its functions by $\kappa\left(p^{\prime} p^{\prime \prime}\right)$ or $\widetilde{\kappa}$.

The process of composition can be extended to more than two classes. In particular we shall use the composite class $\mathfrak{B}^{\prime} \mathfrak{P}^{\prime \prime} \mathfrak{X}$ and the class $\mathfrak{S}$ on $\mathfrak{P}^{\prime} \mathfrak{P}^{\prime \prime} \mathfrak{X}$ to $\mathfrak{A}$, which is defined to be the *-composite of $\mathfrak{M}^{\prime} \mathfrak{M} \mathfrak{M}^{\prime \prime} \mathfrak{S}$, i. e.,

$$
\mathfrak{S}=\left(\mathfrak{M}^{\prime} \mathfrak{M}^{\prime \prime} \mathfrak{S}\right)_{*}=\left(\left(\mathfrak{M}^{\prime} \mathfrak{M}^{\prime \prime} \mathfrak{S}\right)_{L}\right)_{\mathfrak{W}^{\prime}} \mathfrak{M}^{\prime \prime} \mathfrak{E} \text {. }
$$

The elements of this class will be denoted by $\alpha\left(p^{\prime} p^{\prime \prime} x\right)$, or $\alpha$, and $\eta\left(p^{\prime} p^{\prime \prime} x\right)$, or $\eta$.

We have the following propositions on properties and interrelations between the classes $\mathfrak{M Z ^ { \prime }}, \mathfrak{M}^{\prime \prime}, \mathfrak{C}, \mathfrak{\Omega}$, and $\mathfrak{S}$ :

(a) If $\mathfrak{M}^{\prime L C D D_{0}}$ and $\mathfrak{M}^{\prime \prime L C D D_{0}}$, then $\Omega^{L C D D_{0}}$. Any function $\kappa$ of $\Omega^{\prime}$ belongs to $\mathfrak{M}^{\prime}$ for $p^{\prime \prime}$ fixed and to $\mathfrak{M}^{\prime \prime}$ for $p^{\prime}$ fixed.

(b) If $\mathfrak{M}^{\prime L C D D_{0}}$ and $\mathfrak{M}^{\prime \prime}{ }^{L C D D_{0}}$, then $\mathfrak{S}^{L C D D_{0}}$. Any function $\eta$ of $\mathfrak{S}$ belongs to $\Omega$ for $x$ fixed, and to $\subseteq$ for $p^{\prime}$ and $p^{\prime \prime}$ fixed. The class $\Omega$ is a subclass of the class $\mathfrak{S}$. As a matter of fact, every function $\eta$ of the class $\mathfrak{S}$ is uniformly continuous on $\mathfrak{X}$, relatively uniformly as to the class $\Omega,{ }^{*}$ i. e., there exists a $\kappa$ such that, for every $e$, there exists a $d_{e}$ such that if

then

$$
\left|x_{1}-x_{2}\right| \leqq d_{e},
$$

$$
\left|\eta\left(x_{1}\right)-\eta\left(x_{2}\right)\right| \leqq e|\kappa| \text {. }
$$

4. Operators. $\dagger$ We assume that there is present in our theory an operator $J_{p^{\prime \prime} p^{\prime}}$ or $J$, which transforms functions of the class $\bar{\Re}$ into real or complex numbers, i. e., $J$ is on $\widetilde{\Omega}$ to $\mathfrak{A}$. We shall suppose that $J$ has the following properties:

(a) Linearity $\left(J^{L}\right)$, viz., $J\left(a_{1} \breve{\kappa}_{1}+a_{2} \breve{\kappa}_{2}\right)=a_{1} \breve{\kappa}_{1}+a_{2} \breve{\kappa}_{2}$.

(b) Modular Property $\left(J^{M}\right)$, viz., there exists a nowhere negative functional operation $M$ on nowhere negative real-valued functions of $\breve{\Omega}$ such that if

$$
|\kappa| \leqq \kappa_{0}(\geqq 0), \quad \text { then } \quad\left|J_{\kappa}\right| \leqq M_{\kappa_{0}} .
$$

These conditions are sufficient to secure the additional properties contained in the following propositions:

(1) $J_{p^{\prime \prime} p^{\prime}} \eta\left(p^{\prime} p^{\prime \prime} x\right)$ or $J_{21} \eta$ is a function of the class (5.

(2) $J_{q^{\prime \prime} q^{\prime}} \eta_{1}\left(p^{\prime} q^{\prime \prime} x\right) \eta_{2}\left(q^{\prime} p^{\prime \prime} x\right)$ or $J_{23} \eta_{1} \eta_{2}$ is a function of the class $\mathfrak{S}$.

* Cf. E. H. Moore, loc. cit., p. 101.

† Cí. E. H. Moore, Integral Equations, Bulletin of the American Mathem a t i c a I S o c i e t y, vol. 28, pp. 351, 361; Functional Operations, loc. cit., p. 238. 
(3) $J_{p^{\prime \prime} p^{\prime}} J_{q^{\prime \prime} q^{\prime} \kappa_{1}}\left(p^{\prime} q^{\prime \prime}\right) \kappa_{2}\left(q^{\prime} p^{\prime \prime}\right)=J_{q^{\prime \prime} q^{\prime}} J_{p^{\prime \prime} p^{\prime} \kappa_{1}}\left(p^{\prime} q^{\prime \prime}\right) \kappa_{2}\left(q^{\prime} p^{\prime \prime}\right)$ or $J_{41} J_{23} \kappa_{1} \kappa_{2}=J_{23} J_{41} \kappa_{1} \kappa_{2}$, i. e., two successive $J$-operations are commutative.

(4) $J$ is ultra-continuous, i. e., if

$$
L_{n} \eta_{n}=\eta\left(\mathfrak{B}^{\prime} \mathfrak{P}^{\prime \prime} \mathfrak{X} ; \mathfrak{S}\right), \quad \text { then } \quad L_{n} J \alpha \eta_{n}=J \alpha \eta\left(\mathfrak{P}^{\prime} \mathfrak{P}^{\prime \prime} \mathfrak{X} ; \mathfrak{S}\right) \text {. }
$$

As a consequence $J$ is also continuous, i. e., if

$$
L_{n} \kappa_{n}=\kappa\left(\mathfrak{P}^{\prime} \mathfrak{P}^{\prime \prime} ; \Re\right), \quad \text { then } \quad L_{n} J_{\kappa_{n}}=J_{\kappa} .
$$

In addition to the general operator $J$ on $\breve{\Re}$ to $\mathfrak{A}$, we shall make use of a special functional operator, the indefinite integral

$$
\int_{x_{0}}^{x} d x \text { or } I \text {, }
$$

operating on functions of the class $\mathbb{E}$, and yielding functions of a subclass $(\mathfrak{5})$ of this class. On account of the nature of the class $\mathfrak{S}$, the result of applying $I$ to a function this class of yields again a function of $\mathfrak{S E}$, or rather of a subclass of $\mathfrak{S}$, which we shall denote by $\mathfrak{S}^{\prime}$. We shall define the class $\mathfrak{S}^{\prime}$ to be the class of all functions of the form $I \eta+\kappa$. Evidently this class is linear.

The operator $I$ has the properties $L$ and $M$, the modular function being $\int_{a}^{b} d x$. As a consequence, $I$ is also ultracontinuous. Since relative uniformity as to functions of the class $\mathbb{E}$ is equivalent to ordinary uniformity, we have:

(5) If $L_{n} \eta_{n}=\eta\left(\mathfrak{P}^{\prime} \mathfrak{P}^{\prime \prime} \mathfrak{X} ; \mathfrak{R}\right)$ then $L_{n} I \eta_{n}=I \eta\left(\mathfrak{P}^{\prime} \mathfrak{P}^{\prime \prime} \mathfrak{X} ; \Re\right)$.

Further, as in the case of two successive $J$-operations, we show that $I$ and $J$ are commutative on functions of the class $\mathfrak{S}$, i. e.,

(6) For every $\eta$ we have $I J \eta=J I \eta$.

The extension of the operator $I$ to permit of operation on functions of the form $\eta_{1}\left(p^{\prime} p^{\prime \prime} x\right) \eta_{2}\left(q^{\prime} q^{\prime \prime} x\right)$, is apparent. In particular we have an extension of the formula for integration by parts, viz.,

(7) $I\left[I \eta_{1}\left(p^{\prime} p^{\prime \prime} x\right) \cdot I \eta_{2}\left(q^{\prime} q^{\prime \prime} x\right)\right]=\left[\eta_{1}(x)-\eta_{1}\left(x_{0}\right)\right] I \eta_{2}-I \eta_{1} \eta_{2}$.

A direct consequence of this proposition and of proposition (6) is that, if $\eta_{1}$ and $\eta_{2}$ belong to $\mathfrak{S}^{\prime}$, then

(8) $J_{23} \eta_{1} \eta_{2}$ or $J_{q^{\prime \prime} q^{\prime}} \eta_{1}\left(p^{\prime} q^{\prime \prime} x\right) \eta_{2}\left(q^{\prime} p^{\prime \prime} x\right)$ is again a function of $\mathfrak{S}^{\prime}$.

Finally we shall use the operator $d / d x$ or $D_{x}$ or $D$, assuming that it is the inverse of integration $I . \quad D_{x}$ or $D$, then, operates on functions of the class $\mathfrak{S}$ ' and yields functions of the class $\mathfrak{S}$, and in particular:

$$
D(I \eta+\kappa)=\eta \text {. }
$$

Hence if $\eta$ is of class $\mathfrak{S}^{\prime}$, we have

(9) $I D \eta=\eta(x)-\eta\left(x_{0}\right)$.

From the propositions on the operator $I$, we can derive corresponding propositions for the operator $D$. In particular, from (6) follows the commutativity of $D$ and $J$ when operating on functions of the class $\mathfrak{S}$ ', i. e., 
(10) For every function $\eta$ of class $\mathfrak{S}^{\prime}$ we have $D J \eta=J D \eta$.

Further from proposition (7), if $\eta_{1}$ and $\eta_{2}$ belong to $\mathfrak{S C}^{\prime}$, then

(11) $D J_{23} \eta_{1} \eta_{2}=J_{23}\left(\eta_{1} D \eta_{2}+\eta_{2} D \eta_{1}\right)$.

5. Summary. To summarize, then, the contents of the preceding paragraphs, we shall assume as the basis or foundation of our theory the following: $\mathfrak{P}^{\prime}$ and $\mathfrak{P}^{\prime \prime}$, general classes of elements $p^{\prime}$ and $p^{\prime \prime}$.

$\mathfrak{X}$ a class of elements $x$ on the linear interval $a \leqq x \leqq b$.

$\mathfrak{M}^{\prime}$ a class of functions $\mu^{\prime}\left(p^{\prime}\right)$ or $\mu^{\prime}$ on $\mathfrak{P}^{\prime}$ to $\mathfrak{A}$, with the properties $L C D D_{0}$. $\mathfrak{M}^{\prime \prime}$ a class of functions $\mu^{\prime \prime}\left(p^{\prime \prime}\right)$ or $\mu^{\prime \prime}$ on $\mathfrak{B}^{\prime \prime}$ to $\mathfrak{A}$, with the properties $L C D D_{0}$.

$(5$ the class of all continuous functions on $\mathfrak{X}$ to $\mathfrak{A}$.

$\Re$ the class $\left(\mathfrak{M}^{\prime} \mathfrak{M}^{\prime \prime}\right)_{*}$ of functions $\kappa\left(p^{\prime} p^{\prime \prime}\right)$ or $\kappa$. It has the properties $L C D D_{0}$.

$\mathfrak{S}$ the class $\left(\mathfrak{M}^{\prime} \mathfrak{M}^{\prime \prime} \mathfrak{C}\right)_{*}$ of functions $\eta\left(p^{\prime} p^{\prime \prime} x\right)$ or $\eta$. It has the properties $L C D D_{0}$ and contains the class $\Omega$.

$\mathfrak{S}^{\prime}$ the class of all functions of the form $I \eta+\kappa$.

$J$ an operator on $\breve{\AA}$ to $\mathfrak{R}$, with the properties $L$ and $M$.

$I=\int_{x_{0}}^{x}$ on $\mathfrak{S}$ to $\mathfrak{S}^{\prime} . \quad I$ and $J$ are commutative processes.

$D=d / d x$, on $\mathfrak{S}^{\prime}$ to $\mathfrak{S}$. $D$ and $J$ are commutative processes.

II. The differential fQuation and its properties

6. The differential equations. In the theory of a system of linear differential equations of the $n$th order

$$
D_{x} y_{i}(x)=\sum_{j=1}^{n} \alpha_{i j}(x) y_{j}(x) \quad(i=1,2, \cdots, n) .
$$

in which the $\alpha_{i j}(x)$ are a system of $n^{2}$ functions of the class $\mathfrak{S}$ on $\mathfrak{X}$, and the $y_{i}(x)$ are to be determined (they will be of class ( ${ }^{\prime}$ ), we find the following theorems:

ThEоREм I. There exists a unique system of sets of solutions $y_{i}(x)$ of class (5' on $\mathfrak{X}$, which satisfy the initial conditions

$$
y_{i}^{(j)}\left(x_{0}\right)=\delta_{i j},
$$

where $\delta_{i j}$ is the Kronecker $\delta$, $i$. e., zero for $i \neq j$ and unity for $i=j$.

TheoRem II. The determinant formed of this system of solutions $y_{i}^{(j)}(x)$ is nct zero on $\mathfrak{X}$. It has the value

$$
e^{\int_{x_{0}}^{x} \Sigma_{i=1}^{n} \alpha_{i i}(x)}=e^{I \Sigma_{i=1}^{n} \alpha_{i i}} .
$$

Theorem III. The general solution of the system can be written in the form

where the $c_{j}$ are constant.

$$
y_{i}(x)=\sum_{j=1}^{n} c_{j} y_{i}^{(j)}(x),
$$


With a view to generalizing this situation we note* that it is not a set of $n$ solutions which is fundamental, but rather a system or matrix of $n^{2}$ solutions $y_{i j}(x)=y_{i}^{(j)}(x)$. In reality Theorem I might read: There exists a unique system $y_{i j}(x)$ of solutions of the $n^{2}$ equations:

$$
D_{x} y_{i j}(x)=\sum_{k=1}^{n} \alpha_{i k}(x) y_{k j}(x)
$$

which satisfy the initial conditions: $y_{i j}\left(x_{0}\right)=\delta_{i j}$. Then we recall that in the theory of functions of two continuous variables, the expression which plays the rôle of a determinant is the series introduced by Fredholm and generally called a Fredholm determinant, viz,

$$
F_{0}(\kappa)=1+\int_{0}^{1} \kappa(x x) d x+\frac{1}{2 !} \int_{0}^{1} \int_{0}^{1}\left|\begin{array}{l}
\kappa\left(x_{1} x_{1}\right) \kappa\left(x_{1} x_{2}\right) \\
\kappa\left(x_{2} x_{1}\right) \kappa\left(x_{2} x_{2}\right)
\end{array}\right| d x_{1} d x_{2}+\cdots
$$

The finite analog of this determinant, however, is not the determinant of the elements $k_{i j}$ but the determinant of the elements $\delta_{i j}+k_{i j}$. This suggests that in our differential equation we replace $y_{i j}(x)$ by $\delta_{i j}+\bar{y}_{i j}(x)$. This gives

$$
\frac{d \bar{y}_{i j}(x)}{d x}=\alpha_{i j}(x)+\sum_{k=1}^{n} \alpha_{i k}(x) \bar{y}_{k j}(x) \text {. }
$$

Relative to this new equation the three theorems stated above become

THEOREM I. There exists a unique system of solutions $y_{0 i j}(x)$ of class $\sqrt{5}$ on $\mathfrak{X}$ which satisfy the initial conditions $y_{i j}\left(x_{0}\right)=0$.

Theorem II. The analog of the Fredholm determinant, $i$. e., the determinant of $\delta_{i j}+y_{i j}(x)$ of these solutions is not zero on $\mathfrak{X}$. It has the value

$$
e^{I \sum_{i=1}^{n} \alpha_{i i}(x)} .
$$

TheOREM III. The general solution of this system of equations can be uritten in the form

$$
y_{i j}(x)=c_{i j}+y_{0 i j}(x)+\sum_{k=1}^{n} c_{i k} y_{0 k j}(x)
$$

where the $c_{i j}$ are a system of $n^{2}$ constants.

Following Schlesinger $\uparrow$ we obtain by a limiting process analogous theorems in the case in which $i$ and $j$ are replaced by the continuous variables $p$ and $q$ ranging over the interval $0 \leqq p \leqq 1$, and $\sum_{i=1}^{i=n}$ by $\int_{0}^{1}$, the equation being

$$
D_{x} \eta(p, q ; x)=\alpha(p, q ; x)+\int_{0}^{1} \alpha(p, r ; x) \eta(r, q ; x) d r .
$$

\footnotetext{
* Cf. Schlesinger, loc. cit., vol. 24, p. 85. Note that Schlesinger considers monogenic functions of a complex variable $z$, while we are considering functions of the real variable $x$.

$\dagger$ Loc. cit., pp. 85,90-97. The idea of passing to a limit in order to obtain results relative to integro-differential equations was previously pointed out by Volterra. Cf. R e n d i c o n t i d e i Lin ce i, ser. 5, vol. 18 (1909), p. 173.
} 
These two equations and theories being analogous point the way towards considering

or

$$
D \eta\left(p^{\prime} p^{\prime \prime} x\right)=\alpha\left(p^{\prime} p^{\prime \prime} x\right)+J_{q^{\prime \prime} q^{\prime}} \alpha\left(p^{\prime} q^{\prime \prime} x\right) \eta\left(q^{\prime} p^{\prime \prime} x\right)
$$

$$
D \eta=\alpha+J \alpha \eta,
$$

where $p^{\prime}, p^{\prime \prime}$ are on the range $\mathfrak{B}^{\prime} \mathfrak{B}^{\prime \prime}, x$ is on $\mathfrak{X}, \alpha$ and $\eta$ belong to $\mathfrak{S}$, and $J$ is a linear operator, as the fundamental equation of our theory, and it is relative to this equation that we obtain theorems including Theorems $\mathrm{I}^{\prime}, \mathrm{II}^{\prime}$, and III' $^{\prime}$ as special cases. It is desirable to consider also the associated homogeneous equation

$$
D \eta=J \alpha \eta \text {. }
$$

7. The existence theorem. An existence theorem for general differential equations has been developed by Moore. ${ }^{*}$ It would be an easy matter to derive from this general result a theorem which would apply in the case of equations $(A)$ and $(B)$. We prefer, however, to proceed directly. We have the following

Theorem I. There exists a unique solution $\eta_{0}$ of class $\mathfrak{S}^{\prime}$ of the equation

$$
D \eta=\alpha+J \alpha \eta,
$$

which satisfies the initial condition $\eta\left(x_{0}\right)=0$, where $x_{0}$ is any element of the class $\mathfrak{X}$.

The proof depends upon the fact that the differential equation

$$
D \eta=\alpha+J \alpha \eta
$$

with the initial condition $\eta\left(x_{0}\right)=0$ is equivalent to the integral equation $\eta=I \alpha+I J \alpha \eta, I$ being $\int_{x_{0}}^{x} d x$. In fact, if there is a solution of the differential equation with the initial condition, we obtain the integral equation by integrating from $x_{0}$ to $x$, i. e., this solution satisfies the integral equation. On the other hand, if there is a solution of the integral equation of class $\mathfrak{S}$ we must have first of all $\eta\left(x_{0}\right)=0$. Further, on account of the form of the equation, it will be of class $\mathfrak{S}$ '. Hence differentiation is permitted and we regain the differential equation. It is therefore sufficient to show that this integral equation has a unique solution.

Suppose then that $\eta=I \alpha+I J \alpha \eta$ has a solution $\eta_{0}$ of class $\mathfrak{S}^{\prime}$. Then by successive substitution we see that $\eta_{0}$ must also satisfy the equation

$$
\eta_{0}=I \alpha+\sum_{n=1}^{m}(I J \alpha)^{n} I \alpha+(I J \alpha)^{m+1} \eta_{0} .
$$

This suggests that $\eta_{0}$ might be of the form:

$$
\eta_{0}=I \alpha+\sum_{n=1}^{\infty}(I J \alpha)^{n} I \alpha .
$$

${ }^{*}$ Cf. Atti di IV Congresso (Rome), vol. II, pp. 113-114; also lectures at the University of Chicago. 
Let us then determine the nature of the convergence of the series on the right-hand side. We recall that the class $\mathfrak{S}$ is dominated by the class $\Omega$ and this in turn by the class $\mathfrak{M}^{\prime} \mathfrak{M}^{\prime \prime}$, and as a matter of fact, on account of the property $D_{0}$, by nowhere negative real-valued functions of this class. Hence for every $\alpha$ there exists $\mu_{0}^{\prime} \geqq 0, \mu_{0}^{\prime \prime} \geqq 0$ such that $|\alpha| \leqq \mu_{0}^{\prime} \mu_{0}^{\prime \prime}$. It follows then that

and so

$$
|I \alpha| \leqq I|\alpha| \leqq \mu_{0}^{\prime} \mu_{0}^{\prime \prime}\left|x-x_{0}\right|
$$

$$
\left|\alpha\left(p^{\prime} q^{\prime \prime}\right) I \alpha\left(q^{\prime} p^{\prime \prime}\right)\right| \leqq \mu_{0}^{\prime}\left(p^{\prime}\right) \mu_{0}^{\prime \prime}\left(q^{\prime \prime}\right) \mu_{0}^{\prime}\left(q^{\prime}\right) \mu_{0}^{\prime \prime}\left(p^{\prime \prime}\right)\left|x-x_{0}\right| .
$$

That is, since $J$ has the properties $M$ and $L$,

or,

$$
\frac{|J \alpha I \alpha|}{\mu_{0}^{\prime}\left(p^{\prime}\right) \mu_{0}^{\prime \prime}\left(p^{\prime \prime}\right)\left|x-x_{0}\right|} \leqq M \mu_{0}^{\prime} \mu_{0}^{\prime \prime},
$$

$$
|J \alpha I \alpha| \leqq \mu_{0}^{\prime}\left(p^{\prime}\right) \mu_{0}^{\prime \prime}\left(p^{\prime \prime}\right) M \mu_{0}^{\prime} \mu_{0}^{\prime \prime}\left|x-x_{0}\right|
$$

Integration yields

$$
|I J \alpha I \alpha| \leqq(1 /(2 !))\left|x-x_{0}\right|^{2} \mu_{0}^{\prime} \mu_{0}^{\prime \prime} M \mu_{0}^{\prime} \mu_{0}^{\prime \prime} .
$$

Repeating this same process we easily show by induction that

$$
\left|(I J \alpha)^{n} I \alpha\right| \leqq \frac{\left|x-x_{0}\right|^{n+1}}{(n+1) !} \mu_{0}^{\prime} \mu_{0}^{\prime \prime}\left(M \mu_{0}^{\prime} \mu_{0}^{\prime \prime}\right)^{n},
$$

and hence the series $I \alpha+\sum_{n}(I J \alpha)^{n} I \alpha$ is term by term less than the series

$$
\sum_{n=0}^{\infty} \frac{\left|x-x_{0}\right|^{n+1}}{(n+1) !} \mu_{0}^{\prime} \mu_{0}^{\prime \prime}\left(M \mu_{0}^{\prime} \mu_{0}^{\prime \prime}\right)^{n}
$$

which series is convergent on $\mathfrak{P}^{\prime} \mathfrak{P}^{\prime \prime} \mathfrak{X}$ uniformly as to the function $\mu_{0}^{\prime} \mu_{0}^{\prime \prime}$. Hence the series for $\eta_{0}$ is convergent uniformly relative to the class $\mathfrak{M}^{\prime} \mathfrak{M} \mathfrak{R}^{\prime \prime}$ and, on account of the closure of the class $\mathfrak{S}$, represents a function of this class.

The function defined by this series satisfies the original integral equation. For from the relative uniform convergence of the series and the continuity of $I$ and $J$, it follows that term by term integration is permissible, i. e., we have $I J \alpha \eta_{0}=\sum_{n=1}^{n=\infty}(I J \alpha)^{n} I \alpha$, and hence

$$
I J \alpha \eta_{0}+I \alpha=\eta_{0} .
$$

This shows incidentally that $\eta_{0}$ is of class $\mathfrak{S}^{\prime}$.

Finally, the uniqueness of the solution follows from the fact that for every $\eta$ of class $\mathfrak{K}$ we have $L_{n=\infty}(I J \alpha)^{n} \eta=0$, and as a matter of fact relatively uniformly as to the class $\mathfrak{M}^{\prime} \mathfrak{M}^{\prime \prime}$. For from the dominance properties of the class $\mathfrak{F}$ it follows that there exist nowhere negative real-valued functions Trans. Am. Math. Soc. 6 
$\mu_{1}^{\prime}$ and $\mu_{1}^{\prime \prime}$ such that $|\eta| \leqq \mu_{1}^{\prime} \mu_{1}^{\prime \prime}$. By applying the same line of - reasoning as in the case of the convergence of the series for $\eta_{0}$ we show that

$$
\left|(I J \alpha)^{n} \eta\right| \leqq \frac{\left|x-x_{0}\right|^{n}}{n !} \mu_{0}^{\prime} \mu_{1}^{\prime \prime}\left(M \mu_{0}^{\prime \prime} \mu_{0}^{\prime}\right)^{n-1} M \mu_{0}^{\prime \prime} \mu_{1}^{\prime} \text {. }
$$

From which we have

$$
\mathbf{L}_{n=\infty}(I J \alpha)^{n} \eta=0\left(\mathfrak{P}^{\prime} \mathfrak{P}^{\prime \prime} \mathfrak{X} ; \mathfrak{M}^{\prime} \mathfrak{M}^{\prime \prime}\right) \text {. }
$$

8. Excursus on Fredholm determinants.* In the theory of the general linear integral equation of the form

$$
\mu_{1}^{\prime}=\mu_{2}^{\prime}+z J_{\kappa} \mu_{2}^{\prime},
$$

in which $\mu_{1}^{\prime}$ and $\mu_{2}^{\prime}$ belong to $\mathfrak{M}^{\prime}$, $\kappa$ to $\Re$ and $J$ is the same general linear operator which we use above, Moore has defined the Fredholm determinant and the first Fredholm minor as follows:

where

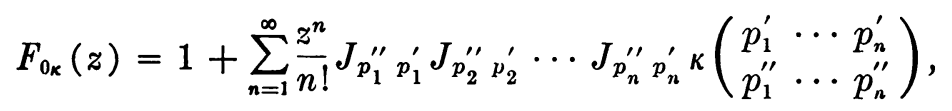

and

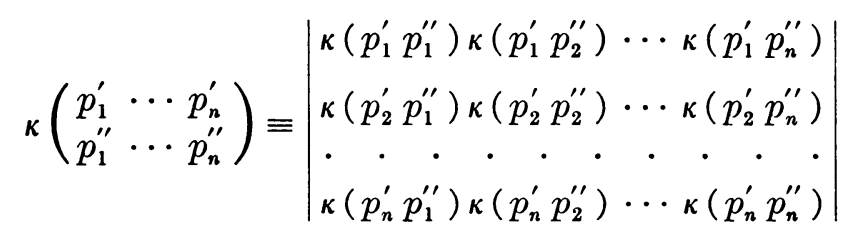

$$
F_{1 \kappa}\left(p^{\prime} p^{\prime \prime} ; z\right)=\kappa\left(p^{\prime} p^{\prime \prime}\right)+\sum_{n=1}^{\infty} \frac{z^{n}}{n !} J_{p_{1}^{\prime \prime}} p_{1}^{\prime} \cdots J_{p_{n}^{\prime \prime}}^{\prime \prime} p_{n}^{\prime} \kappa\left(\begin{array}{cccc}
p^{\prime} & p_{1}^{\prime} & \cdots & p_{n}^{\prime} \\
p^{\prime \prime} & p_{1}^{\prime \prime} & \cdots & p_{n}^{\prime \prime}
\end{array}\right),
$$

and he has shown that the series for $F_{0}$ converges absolutely and uniformly for all finite values of $z$, and that the series for $F_{1}$ converges on $\mathfrak{P}^{\prime} \mathfrak{P}^{\prime \prime}$ absolutely and uniformly as to $\mathfrak{M}^{\prime} \mathfrak{M}^{\prime \prime}$, and uniformly for $z$ finite. $F_{1}$ thus defines a function of the class $\Omega$ for every finite value of $z$. Further we have

$$
F_{1}=F_{0} \kappa-z J_{23} \kappa F_{1}=F_{0} \kappa-z J_{23} F_{1} \kappa,
$$

and as a consequence, if $F_{0} \neq 0$ the equation $\mu_{1}^{\prime}=\mu_{2}^{\prime}+z J_{\kappa} \mu_{2}^{\prime}$ has the unique solution

$$
\mu_{2}^{\prime}=\mu_{1}^{\prime}-z J \frac{F_{1}}{F_{0}} \mu_{1}^{\prime} .
$$

Since for every $x$ of the class $\mathfrak{X}$ the function $\eta$ belongs to the class $\Re$, we can set up these same determinants for $\eta$ and obtain

$$
F_{0 \eta}(x)=1+\sum_{n=1}^{\infty} \frac{1}{n !} J_{p_{1}^{\prime \prime} p_{1}^{\prime}} \cdots J_{p_{n}^{\prime \prime} p_{n}^{\prime} \eta}\left(\begin{array}{l}
p_{1}^{\prime} \cdots p_{n}^{\prime} \\
p_{1}^{\prime \prime} \cdots p_{1}^{\prime \prime}
\end{array} ; x\right)
$$

${ }^{*}$ Cf. E. H. Moore, Integral equations, loc. cit., pp. 353, 354. 
and

$$
F_{1 \eta}(x)=\eta+\sum_{n=1}^{\infty} \frac{1}{n !} J_{p_{1}^{\prime \prime} p_{1}^{\prime}} \cdots J_{p_{n}^{\prime \prime} p_{n}^{\prime} \eta} \eta\left(\begin{array}{cccc}
p^{\prime} & p_{1}^{\prime} & \cdots & p_{n}^{\prime} \\
p^{\prime \prime} & p_{1}^{\prime \prime} & \cdots & p_{1}^{\prime \prime}
\end{array} ; x\right) .
$$

It can be shown that the series for $F_{0 \eta}$ converges uniformly on $\mathfrak{X}$ and hence represents a continuous function on $\mathfrak{X}$; further that the series for $F_{1_{\eta}}$ converges on $\mathfrak{P}^{\prime} \mathfrak{B}^{\prime \prime} \mathfrak{X}$ relatively uniformly as to the class $\mathfrak{S}$ and hence is again a function of this class. In case $\eta$ belongs to the class $\mathfrak{S}^{\prime}$ we can go a step further and assert the

Theorem. If $\eta$ is of class $\mathfrak{S}^{\prime}$ then $F_{0}(x)$ is of class (5', and we have

$$
D_{x} F_{0}(x)=F_{0} J D \eta-J_{41} J_{23}(D \eta) F_{1} \text {. }
$$

By following the line of reasoning used by Fredholm* in the derivation of an analogous equation, we obtain the above expression for $D_{x} F_{0}(x)$, and conclude therefrom that $F_{0}(x)$ is of class $\mathfrak{C}^{\prime}$.

A well-known consequence of this result is the

Theorem. If $\kappa_{1}$ and $\kappa_{2}$ belong to the class $\Omega$ then the Fredholm determinant of $\kappa_{1}+\kappa_{2}+J \kappa_{1} \kappa_{2}$ is equal to the product of the determinants for $\kappa_{1}$ and $\kappa_{2}, i$. e.,

$$
F_{0}\left(\kappa_{1}+\kappa_{2}+J \kappa_{1} \kappa_{2}\right)=F_{0}\left(\kappa_{1}\right) F_{0}\left(\kappa_{2}\right) \text {. }
$$

Since $\eta_{1}$ and $\eta_{2}$ of class $\mathfrak{S}$ for fixed $x$ belong to $\Omega$ it follows that

$$
F_{0}\left(\eta_{1}+\eta_{2}+J \eta_{1} \eta_{2}\right)=F_{0}\left(\eta_{1}\right) F_{0}\left(\eta_{2}\right) .
$$

9. The Fredholm determinant of $\eta_{0}$. Since the function $\eta_{0}$, the solution of equation $(A)$ for which $\eta\left(x_{0}\right)=0$, whose existence was shown in $\S 7$, is of class $\mathfrak{S}^{\prime}$, we can construct the Fredholm determinant of this function and apply the results of the preceding paragraph. We obtain

But

$$
D F_{0 \eta_{0}}=F_{0 \eta_{0}} J D \eta_{0}-J_{41} J_{23}\left(D \eta_{0}\right) F_{1 \eta_{0}} \text {. }
$$

$$
D \eta_{0}=\alpha+J \alpha \eta_{0}
$$

Substituting and making use of the commutativity of successive $J$-operations, we get

$$
D F_{0 \eta_{0}}=F_{0 \eta_{0}} J \alpha-J J \alpha\left(\eta_{0} F_{0 \eta_{0}}-F_{1 \eta_{0}}-J \eta_{0} F_{1 \eta_{0}}\right) \text {. }
$$

But $\eta_{0}, F_{0 \eta_{0}}$, and $F_{1 \eta_{0}}$ are connected by the relation:

Hence

$$
\eta_{0} F_{0 \eta_{0}}-F_{1 \eta_{0}}-J \eta_{0} F_{1 \eta_{0}}=0 \text {. }
$$

$$
D F_{0 \eta_{0}}=F_{0 \eta_{0}} J \alpha \text {. }
$$

Now $F_{0 \eta_{0}}$ is not identically zero in $x$, since, for $x=x_{0}$ it has the value unity.

* Sur une classe d'équations fonctionelles. A c t a M a th e matic a, vol. 27 (1903), pp. 379,380 . 
Hence for the values of $x$ for which $F_{0 \eta_{0}}$ is not zero, we have

$$
\frac{D F_{0 \eta_{0}}}{F_{0 \eta_{0}}}=J \alpha, \quad \text { i. e., } \quad F_{0 \eta_{0}}=e^{I J a} .
$$

We have thus proved

Theorem II. The Fredholm determinant of the solution $\eta_{0}$ of equation $(A)$ is not zero. It has the value $e^{I J a}$.

10. The General solution of equations (A) and (B). By following the line of reasoning indicated by Schlesinger, ${ }^{*}$ we show that any solution $\eta$ of class $\mathfrak{S}^{\prime}$ of equation $(A)$ can be expressed in the form

$$
\eta=\eta_{1}+\eta_{0}+J \eta_{0} \eta_{1}
$$

and thence that $D \eta_{1}=0$; that is, $\eta_{1}$ is independent of $x$ and hence a function of the class $\Omega$. We therefore have:

Theorem III a. The general solution of equation $(A)$ of the class $\mathfrak{S}$ ' can be written in the form

$$
\eta=\cdot \kappa+\eta_{0}+J \eta_{0} \kappa,
$$

where $\kappa$ is a function of the class $\Omega$, and $\eta_{0}\left(x_{0}\right)=0$.

From the second theorem of $\S 8$ it follows that the Fredholm determinant of $\eta$ is the product of the Fredholm determinants of $\kappa$ and $\eta_{0}$ and hence is zero only when the determinant of $\kappa$ is zero. Moreover the form of the general solution together with the fact that the Fredholm determinant of $\eta_{0}$ is not zero yields the more general

Existence Theorem. There exists a unique solution of equation $(A)$ of class $\mathfrak{S}^{\prime}$ which satisfies the initial condition $\eta\left(x_{0}\right)=\kappa_{0}$, where $x_{0}$ is any element of $\mathfrak{X}$ and $\kappa_{0}$ any function of $\Omega$.

We can proceed in a somewhat similar way for the homogeneous equation $(B)$. By observing that the difference of two solutions of the equation $(A)$ is a solution of equation $(B)$ we easily obtain $\dagger$

Theorem III $b$. The general solution of $D \eta=J \alpha \eta$ can be written in the form

$$
\eta=\kappa+J \eta_{0} \kappa
$$

where $\kappa$ is a function of the class $\Omega$.

Observe that this theorem does not give the general solution of equation $(B)$ in terms of a particular solution of the same equation, but in terms of a particular solution of the non-homogeneous equation $(A)$. As in the previous case we can state the

Existence Theorem. There exists a unique solution of equation (B) of class $\mathfrak{S}^{\prime}$ which satisfies the initial condition $\eta\left(x_{0}\right)=\kappa_{0}$, where $x_{0}$ is any element of $\mathfrak{X}$ and $\kappa_{0}$ is any function of $\Re$.

* Loc. cit., pp. 99, 100.

$\dagger$ Cf. Schlesinger, loc. cit., p. 97. 
11. The adjoint equations. In the theory of systems of $n$ linear differential equations, there is associated with the system

the system

$$
D y_{i}(x)=\sum_{j=1}^{n} \alpha_{i j}(x) y_{j}(x)
$$

$$
D z_{i}(x)=-\sum_{j=1}^{n} z_{j}(x) \alpha_{j i}(x)
$$

obtained by changing the sign of the functions $\alpha_{i j}(x)$ and summing with respect to the first of the two subscripts instead of the second. Similarly, in the case where $i$ and $j$ are replaced by continuous variables, Schlesinger* points out that it is desirable to call

$$
D \eta(p, q ; x)=-\alpha(p, q ; x)-\int_{a}^{b} \eta(p, r ; x) \alpha(r, q ; x) d r
$$

the adjoint of the equation

$$
D \eta(p, q ; x)=\alpha(p, q ; x)+\int_{a}^{b} a(p, r ; x) \eta(r, q ; x) d r .
$$

On the basis of these observations we define the equation

$$
D \eta=-\alpha-J \eta \alpha
$$

to be the adjoint of equation $(A)$, and

$$
D_{\eta}=-J_{\eta \alpha}
$$

to be the adjoint of equation $(B)$.

If $\eta$ and $\hat{\eta}$ are any functions of $\mathfrak{S}^{\prime}$, we denote by $M_{1}(\eta)$ the function of $\mathfrak{g}$ represented by the expression $D_{\eta}-\alpha-J \alpha \eta$, and by $N_{1}(\hat{\eta})$ the function represented by $D \hat{\eta}+\alpha+J \hat{\eta} \alpha$. We then have the following formula which is analogous to the Green's Formula,

$$
D(\eta+\hat{\eta}+J \hat{\eta} \eta)=M_{1}(\eta)+N_{1}(\hat{\eta})+J \hat{\eta} M_{1}(\eta)+J N_{1}(\hat{\eta}) \eta
$$

or

$$
(\eta+\hat{\eta}+J \hat{\eta} \eta)_{x_{0}}=I\left(M_{1}(\eta)+N_{1}(\hat{\eta})+J \hat{\eta} M_{1}(\eta)+J N_{1}(\hat{\eta}) \eta\right) .
$$

For by definition we have

$$
\begin{aligned}
J\left(\hat{\eta} M_{1}(\eta)+N_{1}(\hat{\eta}) \eta\right) & =J\left(\hat{\eta} D_{\eta}+(D \hat{\eta}) \eta+\alpha \eta-\hat{\eta} \alpha-\hat{\eta} J \alpha \eta+(J \hat{\eta} \alpha) \eta\right) \\
& =J(D \hat{\eta} \eta)+J \alpha \eta-. J \hat{\eta} \alpha \\
& =J(D \hat{\eta} \eta)-M_{1}(\eta)-\alpha+D \eta-N_{1}(\hat{\eta})+\alpha+D_{\hat{\eta}}
\end{aligned}
$$

or

$$
D \eta+D \hat{\eta}+J D \hat{\eta} \eta=M_{1}(\eta)+N_{1}(\hat{\eta})+J \hat{\eta} M_{1}(\eta)+J N_{1}(\hat{\eta}) \eta .
$$

* Loc. cit., p. 117. 
By using the commutativity of the operators $D$ and $J$ when operating on functions of the class $\mathfrak{S}^{\prime}$ we get the first form given above. The second form is the result of integration between $x_{0}$ and $x$.

Similarly if we denote by $M_{2}(\eta)$ the expression $D_{\eta}-J \alpha \eta$, and by $N_{2}(\hat{\eta})$ the function $D \hat{\eta}+J \hat{\eta} \alpha$, we have

$$
D J \hat{\eta} \eta=J\left(\hat{\eta} M_{2}(\eta)+N_{2}(\hat{\eta}) \eta\right)
$$

or

$$
(J \hat{\eta} \eta)_{x_{0}}^{x}=I J\left(\hat{\eta} M_{2}(\eta)+N_{2}(\hat{\eta}) \eta\right) .
$$

Formula $G_{1}$ is immediately applicable to the question of the solution of the equation $\left(A^{\prime}\right)$. As a matter of fact, suppose $\hat{\eta}_{0}$ is a solution of $\left(A^{\prime}\right)$ for which $\hat{\eta}_{0}\left(x_{0}\right)=0$. If we substitute for $\eta$ the solution $\eta_{0}$ of $(A)$ and for $\hat{\eta}$ this solution $\hat{\eta}_{0}$ of $\left(A^{\prime}\right)$ we have

$$
\eta_{0}+\hat{\eta}_{0}+J \hat{\eta}_{0} \eta_{0}=0 .
$$

On the other hand if $\hat{\eta}_{0}$ is related to $\eta_{0}$ by means of this last equation we have from formula $\left(G_{1}\right)$

$$
\begin{aligned}
M_{1}\left(\eta_{0}\right)+N_{1}\left(\hat{\eta}_{0}\right)+J \hat{\eta}_{0} M_{1}\left(\eta_{0}\right)+J N_{1}\left(\hat{\eta}_{0}\right) & \eta_{0} \\
& =N_{1}\left(\hat{\eta}_{0}\right)+J N_{1}\left(\hat{\eta}_{0}\right) \eta_{0}=0 .
\end{aligned}
$$

But since the Fredholm determinant of $\eta_{0}$ is not zero, this has but one solution

that is, we have

$$
N_{1}\left(\hat{\eta}_{0}\right)=0 \text {, }
$$

Theorem IV. The conditions $N_{1}\left(\hat{\eta}_{0}\right)=0$ with $\hat{\eta}_{0}\left(x_{0}\right)=0$ are equivalent to

$$
\eta_{0}+\hat{\eta}_{0}+J \hat{\eta}_{0} \eta_{0}=0 .
$$

However, this is in the form of a reciprocal relation in the theory of linear integral equations and therefore at once follows

CoRollary I. The solution of equation $\left(A^{\prime}\right)$ which satisfies the initial condition $\hat{\eta}_{0}\left(x_{0}\right)=0$ is

$$
\hat{\eta}_{0}=-\frac{F_{1 \eta_{0}}}{F_{0 \eta_{0}}}
$$

where $F_{0 \eta_{0}}$ and $F_{1 \eta_{0}}$ are respectively the Fredholm determinant and first minor of $\eta_{0}$.

Further since the Fredholm determinant of $\eta_{1}+\eta_{2}+J \eta_{1} \eta_{2}$ is the product of the Fredholm determinants of $\eta_{1}$ and $\eta_{2}$, we have

Corollary II. The Fredholm determinant of the solution $\hat{\eta}_{0}$, for which $\hat{\eta}_{0}\left(x_{0}\right)=0$, is not zero. It has the value $e^{-1 J a}$.

In a manner similar to that used for equations $(A)$ and $(B)$, we prove

THEOREM III'. The general solution of equation $\left(A^{\prime}\right)$ is expressible in the form

$$
\hat{\eta}=\hat{\eta}_{0}+\kappa+J \kappa \hat{\eta}_{0}
$$


and of equation $\left(B^{\prime}\right)$, in the form

$$
\hat{\eta}=\kappa+J_{\kappa} \hat{\eta}_{0} .
$$

For any solution of equation $(A)$ and any solution of $\left(A^{\prime}\right)$ we have

THEOREM IV'. If $\eta$ and $\hat{\eta}$ are any solutions of class $\mathfrak{S}^{\prime}$ of equations $(A)$ and $\left(A^{\prime}\right)$ respectively, then

$$
\eta+\hat{\eta}+J \hat{\eta} \eta=\kappa,
$$

where $\kappa$ is some function of the class $\mathfrak{R}, i . e .$, independent of $x$.

In terms of the solution $\hat{\eta}_{0}$ of the equation $\left(A^{\prime}\right)$ it is an easy matter* to obtain

$$
\eta=\kappa+J \kappa \eta_{0}+I \alpha_{0}+J \eta_{0} I \alpha_{0}+I J \hat{\eta}_{0} \alpha_{0}+J \eta_{0} I J \hat{\eta}_{0} \alpha_{0},
$$

as the general solution for the non-homogeneous equation

$$
D \eta=\alpha_{0}+J \alpha \eta \text {. }
$$

In a similar way, the general solution of the equation

$$
D_{\eta}=\alpha_{0}-J_{\eta \alpha}
$$

is expressible in terms of the solution $\eta_{0}$ of equation $(A)$.

12. Some examples. We digress in this paragraph in order to apply the results of the preceding paragraphs to some special cases.

We consider first of all the case in which the function $\alpha$ is independent of $x$, i. e., $\alpha$ is the same function of the class $\Omega$ for every $x$ of $\mathfrak{X}$. In the solution $\eta_{0}$ of the equation $(A)$ for which $\eta_{0}\left(x_{0}\right)=0$,

$$
\eta_{0}=\sum_{n=0}^{\infty}(I J \alpha)^{n} I \alpha
$$

it will then be possible to carry out the integrations and we obtain

$$
\eta_{0}=\sum_{n=1}^{\infty} \frac{\left(x-x_{0}\right)^{n}}{n !} \alpha^{(n)},
$$

where $\alpha^{(n)}$ is defined as follows:

$$
\alpha^{(n)}=J \alpha^{(n-1)} \alpha \quad \text { and } \quad \alpha^{(1)}=\alpha,
$$

i. e., it is the $n$th iterated kernel of $\alpha$ in the sense of linear integral equations. On account of the properties of the class $\Omega$ and the operator $J$, for every function $\alpha$ there will exist nowhere negative functions $\mu_{0}^{\prime}$ and $\mu_{0}^{\prime \prime}$ and a constant $b$ such that $\alpha^{(n)} \leqq \mu_{0}^{\prime} \mu_{0}^{\prime \prime} b^{n-1}$ as in $\S 7$ above, or in the theory of integral equations. It follows then that the series for $\eta_{0}$ is convergent for all values of $x$, relatively uniformly as to the class $\Re$ on $\mathfrak{P}^{\prime} \mathfrak{P}^{\prime \prime}$, so that $\eta_{0}$ is of class $\mathfrak{W}$ on $\mathfrak{B}^{\prime} \mathfrak{B}^{\prime \prime} \mathfrak{X}$, where $\mathfrak{X}$ may be the class $-\infty<x<\infty$. The same will be

\footnotetext{
${ }^{*}$ Cf. Schlesinger, loc. cit., pp. 119, 120.
} 
true of the function obtained by putting $x_{0}=0$. This function we denote by $\eta(\alpha x)$, i. e.,

In the case in which

$$
\eta(\alpha x)=\sum_{n=1}^{\infty} \frac{x^{n} \alpha^{(n)}}{n !}=\sum_{n=1}^{\infty} \frac{(x \alpha)^{(n)}}{n !} .
$$

$$
\mathfrak{P}^{\prime}=\mathfrak{P}^{\prime \prime}=\mathfrak{P}^{\mathrm{IV}}=[0 \leqq p \leqq 1]
$$

and $\mathfrak{M}^{\prime}=\mathfrak{M}^{\prime \prime}=\mathfrak{M}^{\mathrm{IV}}=$ the class of all continuous functions on $\mathfrak{P}^{\mathrm{IV}}$, Schlesinger has called the function $\eta(\alpha x)$ the Volterra Transcendental.*

An addition theorem for this function $\eta$ is an immediate consequence of the form of the general solution of the equation $(A)$. For evidently

and hence

$$
\eta_{0}(x)=\eta\left[\alpha\left(x-x_{0}\right)\right],
$$

$$
\eta(\alpha x)=\kappa+\eta\left[\alpha\left(x-x_{0}\right)\right]+J_{\eta}\left[\alpha\left(x-x_{0}\right)\right] \kappa .
$$

The function $\kappa$ is determined by letting $x=x_{0}$. This gives $\kappa=\eta\left(\alpha x_{0}\right)$, i. e.,

$$
\eta(\alpha x)=\eta\left(\alpha x_{0}\right)+\eta\left[\alpha\left(x-x_{0}\right)\right]+J \eta\left[\alpha\left(x-x_{0}\right)\right] \eta\left(\alpha x_{0}\right) .
$$

If we let $x=y+z$ and $x_{0}=y$, then

$$
\eta[\alpha(y+z)]=\eta(\alpha y)+\eta(\alpha z)+J \eta(\alpha z) \eta(\alpha y) .
$$

Since $\eta(\alpha 0)=0$, it follows from this that

$$
\eta(\alpha y)+\eta(\alpha(-y))+J_{\eta}[\alpha(-y)] \eta(\alpha y)=0,
$$

i. e., $\eta(\alpha(-y))$ is the reciprocal of $\eta(\alpha y)$, a result which we might expect if we note that the solution of the adjoint equation $D \eta=-\alpha-J_{\eta \alpha}$ for which $\eta(0)=0$ is

$$
\hat{\eta}(x)=\sum_{n=1}^{\infty} \frac{(-\alpha)^{(n)} x^{n}}{n !}=\sum_{n=1}^{\infty} \frac{\alpha^{(n)}(-x)^{n}}{n !}=\eta[\alpha(-x)] .
$$

The function $\eta(\alpha x)$ thus has properties somewhat similar to those of $e^{x}$. As a matter of fact, we can represent $\eta(\alpha x)$ symbolically in the form

$$
\left(e^{a x}-1\right) \text {, }
$$

where $\alpha^{n}$ is to be replaced by $\alpha^{(n)}$. If we note that we obtain from

$$
\left(e^{a_{1} x}-1\right)\left(e^{\alpha_{2} y}-1\right)=J \eta\left(\alpha_{1} x\right) \eta\left(\alpha_{2} y\right),
$$

$$
\begin{aligned}
\left(e^{a_{1} x}-1\right)\left(e^{a_{2} y}-1\right) & =e^{a_{1} x+a_{2} y}-e^{a_{1} x}-e^{a_{2} y}+1 \\
& =\left(e^{a_{1} x+a_{2} y}-1\right)-\left(e^{a_{1} x}-1\right)-\left(e^{a_{2} y}-1\right)
\end{aligned}
$$

${ }^{*}$ Cf. Schlesinger, loc. cit., p. 113; Volterra, Leçons sur les fonctions de lignes, pp. 127, 158 , and 159. 
the more general addition theorem

$$
J \eta\left(\alpha_{1} x\right) \eta\left(\alpha_{2} y\right)=\eta\left(\alpha_{1} x+\alpha_{2} y\right)-\eta\left(\alpha_{1} x\right)-\eta\left(\alpha_{2} y\right),
$$

provided $\alpha_{1}$ and $\alpha_{2}$ are such that

$$
J \alpha_{1} \alpha_{2}=J \alpha_{2} \alpha_{1}
$$

i. e., $\alpha_{1}$ and $\alpha_{2}$ are permutable.*

Another type of interesting results may be obtained by specializing the ranges $\mathfrak{B}^{\prime}$ and $\mathfrak{P}^{\prime \prime}$, the classes of functions $\mathfrak{M}^{\prime}$ and $\mathfrak{M}^{\prime \prime}$, and the operator $J$. We note the following intances:

(a) Linear integro-differential equations. $\dagger$ We take $\mathfrak{P}^{\prime}=\mathfrak{P}^{\prime \prime}=\mathfrak{P}^{\mathrm{IV}}=$ the class of all points on the linear interval $0 \leqq p \leqq 1$. Further let $\mathfrak{M}^{\prime}=\mathfrak{M}^{\prime \prime}$ $=\mathfrak{M}^{\mathrm{IV}}=$ the class of all continuous function on $\mathfrak{P}^{\mathrm{IV}}$. Then $\Re$ is the class of all continuous functions on $\mathfrak{B P}$ and $\mathfrak{S}$ is the class of all continuous functions on $\mathfrak{B P X}$. $\neq$ The operator $J$ we assume to be the definite integral $\int_{0}^{1} d p$. Then our differential equation $(A)$ takes the form

$$
D_{x} \eta(p q x)=\alpha(p q x)+\int_{0}^{1} \alpha(p r x) \eta(r q x) d r .
$$

We conclude at once, then, that there exists a unique solution of this equation, $\eta_{0}(p q x)$, for which $\eta\left(x_{1}\right)=0$. Moreover if we build the ordinary Fredholm determinant for this solution it will not be zero on $\mathfrak{X}$ but will have as its value

$$
e^{I \int_{0}^{1} \alpha(p p x) d p}
$$

(b) Systems of linear integro-differential equations. $\S$ Let $\mathfrak{P}^{\prime}=\mathfrak{P}^{\prime \prime}=$ the composite class $\mathfrak{P}^{\mathrm{II}_{n}} \mathfrak{P}^{\mathrm{IV}}=$ class of all pairs $(i p)$, where $i=1, \cdots, n$, and $0 \leqq p \leqq 1$. We assume that $\mathfrak{M}^{\prime}=\mathfrak{M}^{\prime \prime}=\left(\mathfrak{M}^{I_{n}} \mathfrak{M}^{I^{V}}\right)_{*}=$ the class of all sets of $n$ continuous functions on $\mathfrak{P}^{\mathrm{IV}}$. Then $\Re$ will be the class of all sets of $n^{2}$ continuous functions of two variables on $\mathfrak{P}^{\mathrm{IV}} \mathfrak{P}^{\mathrm{IV}}$ and $\mathfrak{S}$ will be the class of all sets of $n^{2}$ continuous functions of three variables on $\mathfrak{B}^{\mathrm{IV}} \mathfrak{P}^{\mathrm{IV}} \mathfrak{X}$. We take for the operator $J$ the bipartite operator $\sum_{i=1}^{i=n} \int_{0}{ }^{1}$. Then we have for consideration the following system of linear integro-differential equations

$$
D_{x} \eta_{i j}(p q x)=\alpha_{i j}(p q x)+\sum_{k=1}^{n} \int_{0}^{1} \alpha_{i k}(p r x) \eta_{k i}(r q x) d r .
$$

As a consequence of our general existence theorem, this equation has a

* Cf. Volterra, loc. cit., pp. 124, 158 and 159.

$\dagger$ Cf. Schlesinger, loc. cit., pp. $84 \mathrm{ff}$. He considers the case in which the variable $x$ is replaced by the complex variable $z$. The functions $\eta$ are analytic in $z$.

$\ddagger$ Cf. for instance O. Bolza, loc. cit., p. 291.

$\S$ See also $\S 12$ below. 
unique system of solutions for which $\eta_{i j}\left(p q x_{0}\right)=0$. The Fredholm determinant of this system of solutions which can be written in the form

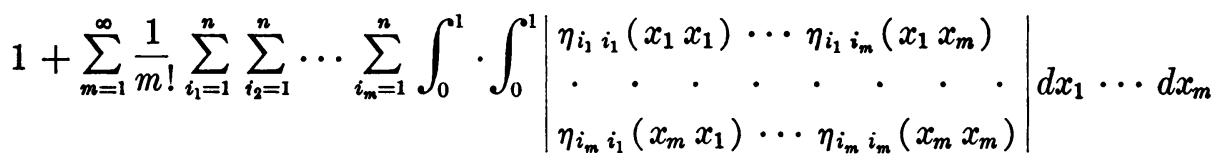

has the value

$$
e^{\Sigma_{i=1}^{n} \int_{0}^{1} I \alpha_{i i}(p p x) d p}
$$

The general solution of the system will be:

$$
\eta_{i j}(p q x)=\kappa_{i j}(p q)+\eta_{0 i j}(p q x)+\sum_{k=1}^{n} \int_{0}^{1} \eta_{0 i k}(p r x) \kappa_{k j}(r q) d r,
$$

where $\kappa_{i j}(p q)$ are a system of continuous functions on $\mathfrak{P P}$.

(c) Infinite systems of differential equations. Let $\mathfrak{P}^{\prime}=\mathfrak{P}^{\prime \prime}=\mathfrak{B}^{\mathrm{III}}$, the class of all positive integers, i. e., $p=1, \cdots n \cdots$. We assume that $\mathfrak{M}^{\prime}=\mathfrak{M}^{\mathrm{II}_{f}}=$ the class of all functions $\mu^{\prime}$ for which $\sum_{p}\left|\mu_{p}\right|^{f}$ is convergent, $f$ being greater than unity, and $\mathfrak{M}^{\prime \prime}=\mathfrak{M}^{\mathrm{II}_{f /(f-1)}}=$ the class of all functions $\mu^{\prime \prime}$ for which

$$
\sum_{p}\left|\mu_{p}^{\prime \prime}\right|^{f /(f-1)}
$$

is convergent. Then $\Omega$ is the class of all functions $\kappa(p q)$ for which there exist $\mu_{p}^{\prime}$ and $\mu_{q}^{\prime \prime}$ so that

$$
|\kappa(p q)| \leqq \mu^{\prime}(p) \mu^{\prime \prime}(q) .^{*}
$$

Further $\mathfrak{S}$ will be the class of all functions which are continuous in $x$, for $p$ and $q$ fixed, and are dominated by a function of the class $\Omega$, i. e., if $\eta(p q x)$ belongs to $\mathfrak{S}$, it will be continuous in $x$, and there will exist a $\kappa(p q)$ such that $|\eta(p q x)| \leqq|\kappa(p q)|$, and conversely. $\dagger$ We assume that the operator $J$ on $\Omega$ is the $\sum_{p=1}^{p=\infty} \kappa(p p)$. That this series actually converges is a result of the inequality: $\ddagger$

$$
\left[\left|\sum_{p=1}^{\infty} \mu^{\prime}(p) \mu^{\prime \prime}(p)\right|\right]^{f} \cong\left[\sum_{p=1}^{\infty}\left|\mu^{\prime}(p)\right|^{f}\right]\left[\sum_{p=1}^{\infty}\left|\mu^{\prime \prime}(p)\right|^{f /(f-1)}\right]^{f-1} .
$$

Since $\Sigma\left|\mu^{\prime}(p)\right|^{f}$ and $\Sigma\left|\mu^{\prime \prime}(p)\right|^{f /(f-1)}$ converge and there exist $\mu^{\prime}(p)$ and $\mu^{\prime \prime}(p)$ such that $|\kappa(p p)| \leqq \mu^{\prime}(p) \mu^{\prime \prime}(p)$, it follows that $\Sigma_{\kappa}(p p)$ will converge also. Evidently $\sum_{p=1}^{p=\infty}$ has the properties $L$ and $M$. We can then

${ }^{*}$ Cf. for instance Bolza, loc. cit., p. 292.

$\dagger$ Cf. Moore, General Analysis, pp. 110-114, 146, 147. This class may also be defined to be the class of all functions which belong to $\Re$ for every $x$ and are continuous in $x$ relatively uniformly as to the class $\Re$. See p. 101 .

‡ Cf. Riesz, Theorie d'équations linéaires à une infinité d'inconnus, p. 45. 
write our differential equation

$$
D \eta(p q x)=\alpha(p q x)+\sum_{r=1}^{\infty} \alpha(p r x) \eta(r q x), \quad p, q=1,2, \cdots, n, \cdots,
$$

and conclude that this infinite system has a unique solution of class $\mathfrak{S}$ ', for which $\eta_{0}\left(p q x_{0}\right)=0$. The Fredholm determinant of this solution reduces to the convergent infinite determinant of

$$
\delta(p q)+\eta_{0}(p q x),
$$

where $\delta(p q)$ is the Kronecker $\delta$, and this determinant has the value

$$
e^{I \Sigma_{p=1}^{p=\infty} \alpha(p p x)}
$$

The general solution of the equation of class $\mathfrak{S}^{\prime}$ is

$$
\eta(p q x)=\kappa(p q)+\eta_{0}(p q x)+\sum_{r} \eta_{0}(p r x) \kappa(r q),
$$

while the general solution of the associated homogeneous equation is

$$
\eta(p q x)=\kappa(p q)+\sum_{r} \eta_{0}(p r x) \kappa(r q),
$$

where $\kappa$ is any function of the class $\Omega$. If in this last case we consider $q$ fixed, we get a function $\phi(p x)$ which belongs to $\mathfrak{M}^{\prime}$ for every $x$. We can consider it a solution of the equation

$$
D \phi(p x)=\sum_{r} \alpha(p r x) \phi(r x) .
$$

If on the other hand we construct the adjoint equation

$$
D_{\eta}(p q x)=-\sum_{r} \eta(p r x) \alpha(r q x)
$$

and fix $p$ in the general solution, we get a function $\psi(q x)$ which belongs to $\mathfrak{M}^{\prime \prime}$ for every $x$, and can be considered a solution of the equation

$$
D \psi(p x)=-\sum_{r} \psi(r x) \alpha(r p x) .
$$

We have here the same phenomenon which we find in the theory of equations of infinitely many variables, where solutions of adjoint equations belong to $\mathfrak{M}^{\mathrm{II}_{f}}$ and $\mathfrak{M}^{\mathrm{II}_{f /(f-1)}}$ respectively.

(d) Finally we consider an instance in which the ranges $\mathfrak{P}^{\prime}$ and $\mathfrak{P}^{\prime \prime}$ are different. Let $\mathfrak{P}^{\prime}=\mathfrak{P}^{\mathrm{II}_{n}}=$ class $i=1,2, \cdots, n$, and $\mathfrak{P}^{\prime \prime}=\mathfrak{P}^{\mathrm{IV}}=$ interval $0 \leqq p \leqq 1$. Let $\mathfrak{M}^{\prime}=\mathfrak{M}^{I^{I_{n}}}=$ class of all $n$-partite numbers, and $\mathfrak{M}^{\prime \prime}=\mathfrak{M}^{\mathrm{IV}}=$ class of all continuous functions on $\mathfrak{P}^{\mathrm{IV}}$. Then $\Re$ will be the class of all sets of $n$ continuous functions on $\mathfrak{B}$, while $\mathfrak{S}$ will be the class of all sets of $n$ continuous functions on $\mathfrak{P X}$. Finally let the operator $J$ on a 
function $\kappa(i, p)$, or $\kappa_{i}(p)$ be

$$
\sum_{i=1}^{n} \int_{0}^{1} \omega_{i}(p) \kappa_{i}(p) d p,
$$

where $\omega_{i}(p)$ belongs to $\Re$.

Then our differential equation becomes

$$
D \eta_{i}(p x)=\alpha_{i}(p x)+\sum_{j=1}^{n} \int_{0}^{1} \alpha_{i}(q x) \omega_{j}(q) \eta_{j}(p x) d q .
$$

We observe that every solution of this equation will also be a solution of the linear integro-differential equation

where

$$
D \eta(p q x)=\alpha(p q x)+\int_{0}^{1} \alpha(p r x) \eta(r q x) d r,
$$

and

$$
\eta(p q x)=\sum_{i=1}^{n} \omega_{i}(p) \eta_{i}(q x)
$$

$$
\alpha(p q x)=\sum_{i=1}^{n} \omega_{i}(p) \alpha_{i}(q x),
$$

and also of the linear system of equations

where

$$
D \eta(i j x)=\alpha(i j x)+\sum_{k=1}^{n} \alpha(i k x) \eta(k j x),
$$

and

$$
\eta(i j x)=\int_{0}^{1} \eta_{i}(p x) \omega_{j}(p) d p
$$

$$
\alpha(i j x)=\int_{0}^{1} \alpha_{i}(p x) \omega_{j}(p) d p .
$$

The converse is not true, excepting when $\omega$ satisfies certain conditions.

Applying our general existence theorem, we conclude that there will exist for our equation a unique solution $\eta_{i}(p x)$ for which $\eta_{i}\left(p x_{0}\right)=0$. When we build the expression corresponding to the Fredholm determinant of this solution with the operator defined above, we find that it may be regarded as the Fredholm determinant of $\eta(p q x)=\sum_{i=1}^{i=n} \omega_{i}(p) \eta_{i}(q x)$, or as the ordinary determinant of $\delta(i j)+\eta(i j)$ where

Its value will be

$$
\eta(i j)=\int_{0}^{1} \eta_{i}(p x) \omega_{j}(p) d p .
$$

$$
e^{I \int_{0}^{1} \eta(p p x) d p}=e^{I \Sigma_{i=1}^{n} \eta(i i x)}=e^{I \int_{0}^{1} \Sigma_{i=1}^{n} \alpha_{i}(p x) \omega_{i}(p) d p}
$$

13. On systems of linear differential equations. The results of $\S \S 6-10$ 
can be easily extended to the case of a system of equations. As a matter of fact a simple transformation reduces the consideration of a system to that of a single equation.

Suppose then that in place of the class $\mathfrak{P}^{\prime}$ we have $n$ classes $\mathfrak{B}_{i}^{\prime}(i=1, \cdots$, $n)$, and instead of the class $\mathfrak{B}^{\prime \prime}$ we have $n$ classes $\mathfrak{P}_{i}^{\prime \prime}(i=1, \cdots, n)$ which are not necessarily all distinct. Suppose further that in place of $\mathfrak{M}^{\prime}$ on $\mathfrak{B}^{\prime}$ to $\mathfrak{A}$ we have $n$ classes $\mathfrak{M}_{i}^{\prime}$ on $\mathfrak{B}_{i}^{\prime \prime}$ to $\mathfrak{A}$, and in place of $\mathfrak{M}^{\prime \prime}$ on $\mathfrak{P}^{\prime \prime}$ to $\mathfrak{A}$ we have $n$ classes $\mathfrak{M}_{i}^{\prime \prime}$ on $\mathfrak{B}_{i}^{\prime \prime}$ to $\mathfrak{A}$, concerning each of which it will be assumed that they have the properties $L C D D_{0}$. From these classes we construct the $n^{2}$ classes $\mathfrak{R}_{i j}=\left(\mathfrak{M}_{i}^{\prime} \mathfrak{M}_{j}^{\prime \prime}\right)_{*}$ on $\mathfrak{P}_{i}^{\prime} \mathfrak{P}_{j}^{\prime \prime}$ to $\mathfrak{A}$, and the $n^{2}$ classes $\mathfrak{S}_{i j}=\left(\mathfrak{M}_{i}^{\prime} \mathfrak{\mathfrak { M } _ { j } ^ { \prime \prime }} \mathfrak{S}\right)_{*}$ on $\mathfrak{B}_{i}^{\prime} \mathfrak{P}_{j}^{\prime \prime} \mathfrak{X}$ to $\mathfrak{A}$. We replace finally the operator $J$ on $\mathfrak{\Omega}$ to $\mathfrak{A}$ by the $n$ operators $J_{i}$ on $\widetilde{\Omega}_{i i}$ to $\mathfrak{A}$, each of which will be supposed to have the properties $L$ and $M$. We consider then in place of the equation $(A)$ the system of equations

$$
D \eta_{i j}=\alpha_{i j}+\sum_{k=1}^{n} J_{k} \alpha_{i k} \eta_{k j}
$$

and the homogeneous system associated with it

$$
D \eta_{i j}=\sum_{k=1}^{n} J_{k} \alpha_{i k} \eta_{k j}
$$

In treating the corresponding extension in the general theory of linear integral equations, Moore* has suggested a process, viz., that of adjunctional composition which reduces the system of equations to a single equation. This same process is applicable here. We assume that the classes $\mathfrak{P}_{i}^{\prime}$ have no elements in common, a situation which can always be attained by a suitable transformation. We make the same assumption in the case of the classes $\mathfrak{P}_{j}^{\prime \prime}$. Then we define the class $\mathfrak{P}^{\prime}$ to be the class of all elements belonging to any of the $\mathfrak{P}_{i}^{\prime}$, i. e., $\mathfrak{P}^{\prime}=\sum_{i=1}^{i=n} \mathfrak{P}_{i}^{\prime}$, and $\mathfrak{P}^{\prime \prime}$ to be the class of all elements belonging to any of the $\mathfrak{B}_{j}^{\prime \prime}$, i. e. $\mathfrak{P}^{\prime \prime}=\sum_{j=1}^{j=n} \mathfrak{P}_{j}^{\prime \prime}$. The class $\mathfrak{B}^{\prime} \mathfrak{P}^{\prime \prime}$ will be the totality of elements in $\mathfrak{P}_{i}^{\prime} \mathfrak{P}_{j}^{\prime \prime}$. The class $\Re$ will consist of all functions $\kappa$ for which we have

$$
\kappa\left(p_{i}^{\prime} p_{j}^{\prime \prime}\right)=\kappa_{i j}\left(p_{i}^{\prime} p_{j}^{\prime \prime}\right)
$$

and the class $\mathfrak{S}$ will consist of all functions for which we have

$$
\eta\left(p_{i}^{\prime} p_{j}^{\prime \prime} x\right)=\eta_{i j}\left(p_{i}^{\prime} p_{j}^{\prime \prime} x\right) \text {. }
$$

Further we define $J$ operating on a function $\breve{\kappa}$ to be

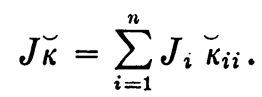

Then the classes $\Re$ and $\mathfrak{S}$ and the operator $J$ will have the properties enum-

* Cf. E. H. Moore, Integral Equations, loc. cit., pp. 355-357. 
erated in $\S \S 3-5$, and our system of differential equations reduces to the single equation

$$
D \eta=\alpha+J \alpha \eta,
$$

while the system $\left(B_{n}\right)$ becomes

$$
D \eta=J \alpha \eta .
$$

By applying the theorems of $\S \S 6-10$, and replacing $\alpha, \eta$, and $J$ by the expressions from which they were derived, we get results of the following type:

Theorem I. The system of equations $\left(A_{n}\right)$ has a unique set of solutions $\eta_{i j}$ which satisfy the initial conditions $\eta_{i j}\left(x_{0}\right)=0$.

Theorem II. The Fredholm determinant of this set of solutions is not zero on $\mathfrak{X}$. It has the value

$$
e^{I \Sigma_{i=1}^{i=n} J_{i} \alpha_{i i}} .
$$

The Fredholm determinant of a set of functions $\eta_{i j}$ would take the form

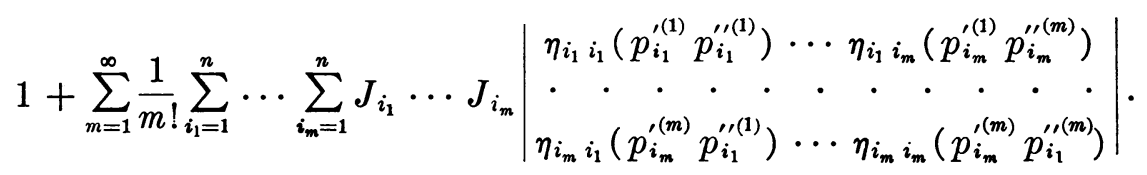

TheOREM III. The general solution of the system $\left(A_{n}\right)$ is

$$
\eta_{i j}=\kappa_{i j}+\eta_{0 i j}+\sum_{k=1}^{n} J_{k} \eta_{0 i k} \kappa_{k j},
$$

where $\kappa_{i j}$ is any function of $\Omega_{i j}$ and $\eta_{0 i j}$ is the solution for which

$$
\eta_{0 i j}\left(x_{0}\right)=0 \text {. }
$$

The general solution of the associated system $\left(B_{n}\right)$ is

$$
\eta_{i j}=\kappa_{i j}+\sum_{k=1}^{n} J_{k} \eta_{0 i k} \kappa_{k j} .
$$

The adjoint equations of $\left(A_{n}\right)$ and $\left(B_{n}\right)$ are

$$
D \eta_{i j}=-\alpha_{i j}-\sum_{k=1}^{n} J_{k} \eta_{i k} \alpha_{k j}
$$

and

$$
D \eta_{i j}=-\sum_{k=1}^{n} J_{k} \eta_{i k} \alpha_{k j} .
$$

If we denote by $\hat{\eta}_{0 i j}$ the set of solutions of equations $\left(A_{n}^{\prime}\right)$ for which

then follows

$$
\hat{\eta}_{0 i j}\left(x_{0}\right)=0 \text {, }
$$

Theonem IV. The functions $\eta_{0 i j}$ and $\hat{\eta}_{0 i j}$ satisfy the reciprocal relations

$$
\eta_{0 i j}+\hat{\eta}_{0 i j}+\sum_{k=1}^{n} J_{k} \hat{\eta}_{0 i k} \eta_{0 k j}=0 .
$$


If we denote by $M_{2 i j}(\eta)$ the functions of $\mathfrak{S}_{i j}$ obtained by substituting a set of functions $\eta_{i j}$ in the expression

and similarly let

$$
D \eta_{i j}-\sum_{k=1}^{n} J_{k} \alpha_{i k} \eta_{k j}=M_{2 i j}(\eta)
$$

$$
D \hat{\eta}_{i j}+\sum_{k=1}^{n} J_{k} \hat{\eta}_{i k} \alpha_{k j}=N_{2 i j}(\hat{\eta})
$$

then we get the Green's Formula:

$$
I \sum_{k=1}^{n} J_{k}\left(\hat{\eta}_{i k} M_{2 k j}(\eta)+N_{2 i k}(\hat{\eta}) \eta_{k j}\right)=\left(\sum_{k=1}^{n} J_{k} \hat{\eta}_{2 k} \eta_{k j}\right)_{x=x_{0}}^{x=x} .
$$

14. Mixed integro-differential equations. An interesting application of the results of the previous paragraph is to the case of mixed differential equations. * Suppose that instead of the single operator $J$ on $\widetilde{\kappa}$ we have $n$ operators

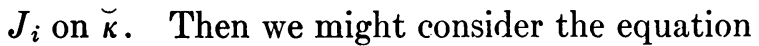

$$
D \eta=\sum_{i=1}^{n} J_{i} \alpha_{i} \eta
$$

If we assume that $\eta_{i j}=\eta$ and $\alpha_{i j}=\alpha_{j}$ for every $i$, then this equation repeated $n$ times could be considered as a system $\left(B_{n}\right)$

$$
D \eta_{i j}=\sum_{k=1}^{n} J_{k} \alpha_{i k} \eta_{k j}
$$

and as a consequence the associated system would take the form

$$
D \eta_{i j}=\alpha_{i j}+\sum_{k=1}^{n} J_{k} \alpha_{i k} \eta_{k j}
$$

The existence theorem applied to this equation reads:

Theorem I. There exists a unique set of solutions of equations $\left(\dot{A}_{1}\right)$ which are such that $\eta_{0 i j}\left(x_{0}\right)=0$. On account of the fact that the system $\left(A_{1}\right)$ really contains only $n$ distinct equations there will be at most $n$ distinct solutions $\eta_{0 j}$.

Theorem II. The Fredholm determinant of the $\eta_{0 j}$ which has the form:

$$
1+\sum_{m=1}^{\infty} \frac{1}{m !} \sum_{i_{1}=1}^{n} J_{i_{1}} \cdots \sum_{i_{m}=1}^{n} J_{i_{m}}\left|\begin{array}{ccccc}
\eta_{i_{1}}\left(p_{1}^{\prime}\right. & \left.p_{1}^{\prime \prime}\right) & \cdots & \eta_{i_{1}}\left(p_{1}^{\prime}\right. & \left.p_{m}^{\prime \prime}\right) \\
\cdot & \cdot & \cdot & \cdot & \cdot \\
\eta_{i_{m}}\left(p_{m}^{\prime}\right. & \left.p_{1}^{\prime \prime}\right) & \cdots & \eta_{i_{m}}\left(p_{m}^{\prime}\right. & \left.p_{m}^{\prime \prime}\right)
\end{array}\right|
$$

is not zero. Its value is $e^{I \Sigma_{i=1}^{i=n} J_{i} \alpha_{i}}$.

Theorem III. The general solution of equation $\left(B_{1}\right)$ is

$$
\eta=\kappa+\sum_{i=1}^{n} J_{i} \eta_{0 i} \kappa .
$$

\footnotetext{
* Cf. E. H. Moore, Integral Equations, loc. cit., p. 358.
} 
The adjoint of the system $(A)$ would be:

$$
D \eta_{i j}=-\alpha_{i j}-\sum_{k=1}^{n} J_{k} \eta_{i k} \alpha_{k j} .
$$

Since however $\alpha_{i j}=\alpha_{j}$ for every $i$, we could drop the first subscripts and get the system of $n$ equations

$$
D \eta_{j}=-\alpha_{j}-\sum_{k=1}^{n} J_{k} \eta_{k} \alpha_{j}
$$

It follows that the adjoint of the single equation $\left(B_{1}\right)$ is the system*

$$
D \eta_{j}=-\sum_{k=1}^{n} J_{k} \eta_{k} \alpha_{j}
$$

ThEOREM IV. Between the solutions $\eta_{0 i}$ of the equation $\left(A_{1}\right)$ and $\hat{\eta}_{0 j}$ of the equations $\left(A_{1}^{\prime}\right)$ for which $\eta_{0 i}\left(x_{0}\right)=0$ and $\hat{\eta}_{0 j}\left(x_{0}\right)=0$, we have the $n$ relations

If we let

$$
\eta_{0 i}+\hat{\eta}_{0 i}+\sum_{k=1}^{n} J_{k} \hat{\eta}_{0 k} \eta_{0 i}=0 \quad(i=1,2, \cdots, \eta) .
$$

and

$$
M(\eta)=D \eta-\sum_{i=1}^{n} J_{i} \alpha_{i} \eta
$$

we have the Green's Formula:

$$
N_{i}(\hat{\eta})=D \hat{\eta}_{i}+\sum_{j=1}^{n} J_{j} \hat{\eta}_{j} \alpha_{i}
$$

$$
I \sum_{i=1}^{n} J_{i}\left(\hat{\eta}_{i} M(\eta)+N_{i}(\hat{\eta}) \eta\right)=\left(\sum_{i=1}^{n} J_{i} \hat{\eta}_{i} \eta\right)_{x=x_{0}}^{x=x} .
$$

We obtain further interesting results if we apply the theorems of this paragraph to the mixed linear integro-differential equation

$$
D \eta(p q x)=\sum_{i=1}^{n} \alpha_{i}(p x) \eta\left(p_{i} q x\right)+\int_{0}^{1} \alpha(p r x) \eta(r q x) d r,
$$

in which we suppose that the ranges $\mathfrak{P}^{\prime}=\mathfrak{P}^{\prime \prime}=\mathfrak{P}^{\mathrm{IV}}=[0 \leqq p \leqq 1], \alpha_{i}(p x)$ belong to the class of all continuous functions on $\mathfrak{B X}$, and $\alpha(p q x)$ to the class of continuous functions on $\mathfrak{B P X}$, while $p_{i}$ are a set of special values of the range $\mathfrak{P}$.

AnN Arbor, Mich., April, 1916

${ }^{*}$ Cf. W. A. Hurwitz, Mixed linear integral equations of the first order, these T r a n a a ct i o n s, vol. 16 (1915), p. 121, where a similar situation occurs. The above seems to show why the adjoint of a single mixed equation is a system. 